\title{
A Design Consideration for Optical Power Dividers Composed of Three Coupled Waveguides
}

\author{
Masashi Hotta, Member, IEEE, Masahiro Geshiro, Member, IEEE, \\ Tomoyuki Arashiba, and Shinnosuke Sawa, Member, IEEE
}

\begin{abstract}
In the present paper, we propose a design consideration for optical power dividers utilizing the distributed coupling among three adjacent slab waveguides. This type of power divider can easily be fabricated by means of the process of ion-exchange. However, being large in scale is a serious problem. We clarify that the size of the device can be remarkably reduced by introducing nonuniformly distributed coupling which is caused by step-like gap variations between the waveguides. In order to obtain wide separations at the output end, we also introduce the low-loss bent structure which is designed by the method of suppression of the optical wave undulation along the waveguide. The numerical results show that low-loss wide separations of optical waves are possible and over a 35\% reduction in device length can be achieved. The numerical analysis is based on the two-dimensional Finite Difference Method.
\end{abstract}

\section{INTRODUCTION}

$\mathbf{L}$ OW-LOSS compact optical power dividers are key components for optical communication systems, optical integrated circuits, and so on. We have investigated so far low-loss design considerations for mode-conversion type optical $Y$ branching waveguides both experimentally and theoretically [1]-[3]. However, some experimental studies have suggested that the $Y$-branches fabricated by the process of ion-exchange suffer scattering losses more than $1 \mathrm{~dB}$ at the branching point [4]

On the contrary, optical power dividers of distributed coupler type, which can also be fabricated easily by the process of ion-exchange, have been proposed as a candidate of forkfree structure [5]-[10]. Although being free from the scattering losses, these dividers have a serious problem of becoming quite large in scale because of their power dividing mechanism. In this paper, we propose a design consideration for low-loss and compact structures utilizing the nonuniformly distributed coupling among three adjacent slab waveguides which is introduced via step-like axial displacement of the outer waveguides. Furthermore, in order to obtain wide separations at the output end, we also introduce low-loss bent structures which are designed with the method of suppression of the optical wave undulation along the waveguide [11].

Manuscript received December 29, 1992; revised September 23, 1993. M. Hotta is with the Department of Electrical and Electronic Engineering, Ehime University, Matsuyama, Ehime 790, Japan.

M. Geshiro is with the Department of Electrical and Electronic Systems, University of Osaka Prefecture, Sakai, Osaka 593, Japan.

T. Arashiba is with the Tyagoku Electric Power Company, Inc., Hiroshima 731-02, Japan.

S. Sawa is with the Department of Electrical Engineering, the University of Osaka Prefecture, Sakai, Osaka 593, Japan.

IEEE Log Number 9402376.
In practical applications, guided-wave devices have been achieved in three-dimensional structures. Most of them can be, however, reduced to two-dimensional ones in good approximation by means of the effective refractive index method. Therefore, two-dimensional slab-waveguide models are studied in the present paper, and thus the two-dimensional (2D) finite difference method (FDM) is employed in the numerical analysis [12].

\section{POWER DIVIDERS COMPOSED OF THREE COUPLED WAVEGUIDES}

\section{A. Uniformly Distributed Coupler}

At first, we consider a power divider of uniformly distributed coupler type composed of three adjacent slab waveguides arranged with a constant interval $s_{3}$ [9], [10], which is shown by the dashed line in Fig. 1. It is assumed that the refractive indices of the film and substrate regions are $n_{F}=1.5115$ and $n_{S}=1.51$, respectively. The waveguide width is also assumed to be $4 \mu \mathrm{m}$ so that each waveguide composing the power divider supports only the dominant mode at an operating wavelength $\lambda=0.6328 \mu \mathrm{m}$. The device length $L$ coincides with the coupling length of the distributed coupler. Therefore, the optical wave incident on the waveguide $A$ is completely transferred to the outer waveguides $B$ and $C$ at $z=L$ where the waveguide $A$ is clipped off. As the interval $s_{3}$ between the waveguides becomes narrower, the coupling among the waveguides is intensified, which leads to reduction in the coupling length. On the other hand, the radiation losses caused by the modal conversion increase considerably with the decreasing interval. In addition to this point, the coupling between the waveguides $B$ and $C$ in the region of $z>L$, which we had better suppress completely, would remain in significant magnitude. Taking these conditions into consideration, we set the interval between the waveguides as $s_{3}=5 \mu \mathrm{m}$.

Fig. 2 shows the field distribution along the power divider. Equidistant grids at intervals of $0.1 \mu \mathrm{m}$ along both the $x$ and $z$ directions are employed throughout the numerical calculations in the present FDM analysis [12]. It is found from the figure that the optical wave incident on the waveguide $A$ is smoothly transferred to the waveguides $B$ and $C$. In this example, the device length $L$ becomes $3899.4 \mu \mathrm{m}$ and the radiation losses due to the modal conversion are almost negligible.

\section{B. Nonuniformly Distributed Coupler of Three Section Structure}

In order to shorten the device length, we introduce a nonuniformly distributed coupler [8] which is shown by the 


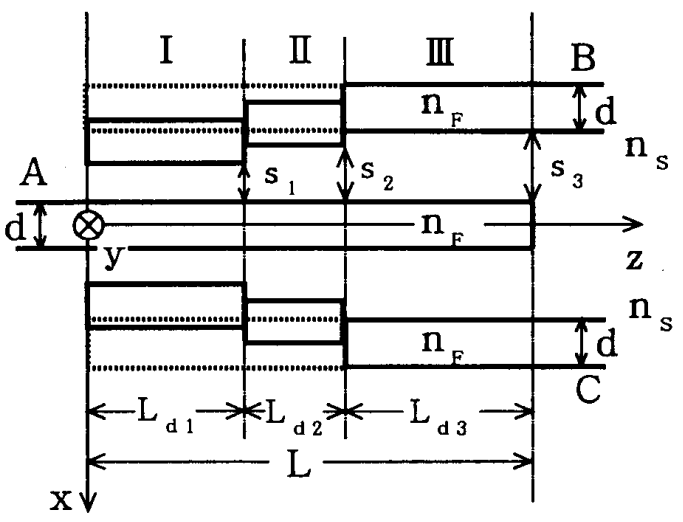

Fig. 1. Optical power dividers utilizing distributed coupling among three adjacent slab waveguides

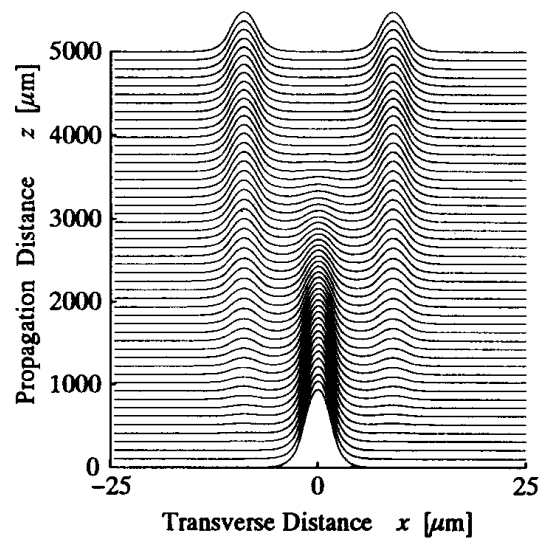

Fig. 2. Field distribution along the optical power divider utilizing uniformly distributed coupling, where $s_{3}=5 \mu \mathrm{m}$ and $\lambda=0.6328 \mu \mathrm{m}$.

solid line in Fig. 1. The whole structure is composed of three sections along the propagation axis: the region I with an interval $s_{1}$, the region II with an interval $s_{2}$, and the region III with an interval $s_{3}$. The waveguide parameters are the same as those of the former coupler. The interval $s_{3}$ between the waveguides in the region III is set as $s_{3}=5 \mu \mathrm{m}$ in order to leave the coupling condition unchanged in the region of $z>L$ for both the uniform and nonuniform couplers. The intervals between the waveguides in the regions $\mathrm{I}$ and II are chosen as $s_{1}=3 \mu \mathrm{m}$ and $s_{2}=4 \mu \mathrm{m}$, respectively, so that the coupling is loosened step-by-step along the structure. Tight coupling around the input end shortens the coupling length and loose coupling around the output end eases the serious dependence of the coupling length on the interval between the waveguides.

Fig. 3 shows the coupling length and normalized radiation losses at the output end relative to the incident power as a function of the length $L_{d 1}$ of the region $I$, where the length of the region II is fixed as $L_{d 2}=200.0 \mu \mathrm{m}$. This figure indicates that as $L_{d 1}$ becomes large, the coupling length reduces, whereas the radiation loss increases. Particularly, the rate of increase in radiation losses rises around $L_{d 1}=715.0$ $\mu \mathrm{m}$. For this reason, we set the length of the region $\mathrm{I}$ as $L_{d 1}=715.0 \mu \mathrm{m}$.

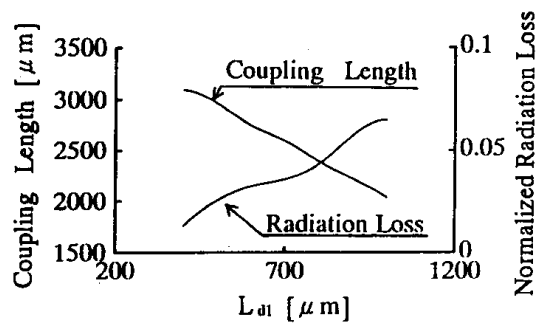

Fig. 3. Coupling length and normalized radiation loss versus $L_{d 1}$ in the nonuniform coupler, where $L_{d 2}=200.0 \mu \mathrm{m}$ and $\lambda=0.6328 \mu \mathrm{m}$.

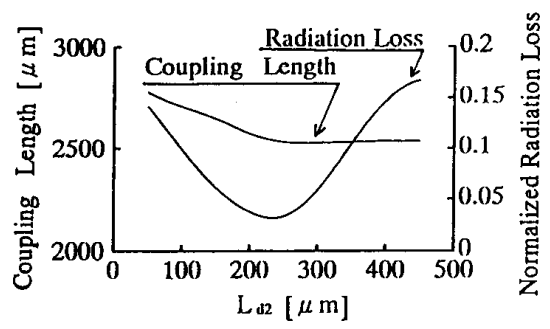

Fig. 4. Coupling length and normalized radiation loss versus $L_{d 2}$ in the nonuniform coupler, where $L_{d 1}=715.0 \mu \mathrm{m}$ and $\lambda=0.6328 \mu \mathrm{m}$.

The functional dependence of the coupling length and normalized radiation losses on $L_{d 2}$ is shown in Fig. 4, where $L_{d 1}=715.0 \mu \mathrm{m}$. The coupling length decreases monotonously as expected with the increase in $L_{d 2}$. It is, however, noticeable that the radiation loss takes the minimum value at $L_{d 2}=236.0 \mu \mathrm{m}$. It has been reported for the case of isolated curved slab waveguides that the well-adjusted abrupt bends offer coherent coupling, by which radiation losses can be reduced remarkably [13]. The characteristics of the radiation losses in Fig. 4 suggest that similar phenomena could be expected also in the present three section structure.

Fig. 5 shows the field distributions along the optical power divider utilizing nonuniformly distributed coupling, where $L_{d 1}=715.0 \mu \mathrm{m}$ and $L_{d 2}=236.0 \mu \mathrm{m}$. This figure illustrates that the optical wave incident on the waveguide $A$ is smoothly transferred to the outer waveguides $B$ and $C$. The device length is estimated to be $L=2537.4 \mu \mathrm{m}$. This means that, with keeping the radiation losses less than $4 \%$, over a $35 \%$ reduction in dimensions can be achieved by introducing the three section structure. If a higher level of radiation losses is allowed, further reduction in the coupling length would be possible.

On the other hand, the uniformly distributed coupler with a device length of $2537.4 \mu \mathrm{m}$ does not suffer any significant radiation losses. However, a $35 \%$ reduction in the coupling length of the uniform coupler reduces the period of a sinusoidal interchange of optical power between the waveguides by the same percentage. This implies some deterioration in tolerance for fabrication errors. Here we attach the greatest importance to the coupling condition at the output end of the coupler. This is the main reason why the uniformly distributed coupler with a interval of $s_{3}=5 \mu \mathrm{m}$ is used as the basis for the comparison to the three section structure.

By the way, one might expect that a nonuniformly distributed coupler with a gradual change of coupling would 


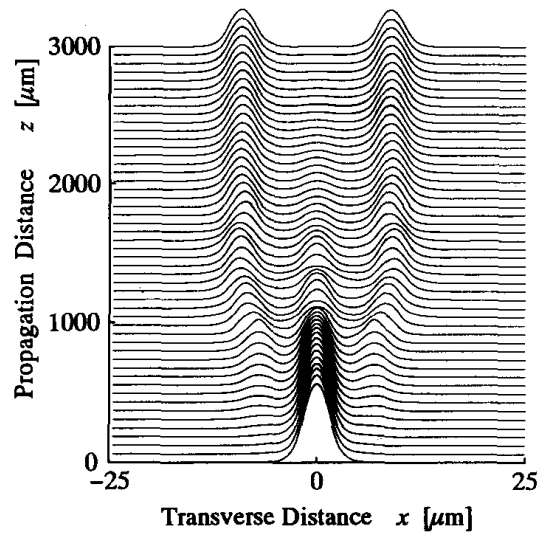

Fig. 5. Field distribution along the optical power divider utilizing nonuniformly distributed coupling, where $L_{d 1}=715.0 \mu \mathrm{m}$ and $\lambda=0.6328 \mu \mathrm{m}$.

be another candidate for better performance. In this case, however, there exist so many parameters to be decided to optimize the structure. Especially, it is very difficult to obtain the structural parameters which give the complete power transfer from the center waveguide to the outer ones. As an examples, we have studied a coupler with a linearly tapered gap which have the same intervals at both input and output ends as the three section structure. We have obtained the optimum coupler length of about $2200 \mu \mathrm{m}$ which gives the maximum output power of $96 \%$. On the other hand, the three section structure presented here is a very simple structure which can easily be optimized. Fig. 3 shows that the three section structure can be reduced to a comparable size if the same amount of radiation loss is allowed. These results signify that the gradually changing coupling does not necessarily offer better performance.

\section{LOW-LOSS DESIGN CONSIDERATION}

It is necessary to adopt bent structures to obtain a wider separation between the waveguides at the output end which may frequently be a crucial requirement. Here we discuss low-loss structures including the bent waveguides designed by means of the method of suppression of the optical wave undulation along the waveguide [11].

\section{A. Low-Loss Multi Bent Structure}

The method of suppression of the wave undulation was originally proposed for low-loss designing by means of the Beam Propagation Method [11], [14]. In practice, this method is applicable to another analytical method for wave propagation problems by which the field distribution along the waveguide can be obtained directly. In this paper, we design low-loss structures by means of the FDM [12] incorporated with the method of suppression of the wave undulation.

Let's summarize the method first. Fig. 6 shows a multi bent and a simple bent structure. The locus of the peak of the field intensity in the simple bent waveguide is also shown in the figure. The locus coinciding with the center axis of the incoming waveguide begins undulating around it after the break. This undulation is caused by the modal conversion

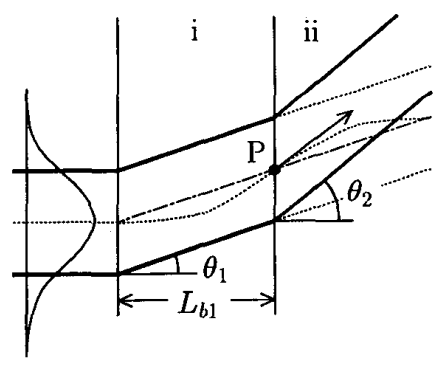

Fig. 6. Undualations of the optical wave along a bent waveguide.

TABLE I

Transmitted Power Versus Slope of the Waveguide in the Region $i i$ WHERE $\lambda=0.6328 \mu \mathrm{m}$ AND (A) $2 h=50.0 \mu \mathrm{m}$ AND (B) $2 h=130.0 \mu \mathrm{m}$.

\begin{tabular}{c|c|c|c|c|c}
\hline$\theta_{3}\left[^{\circ}\right]$ & 0.25 & 0.5 & 0.75 & 1.0 & 1.25 \\
\hline$L_{b 3}[\mu \mathrm{m}]$ & 206.4 & 221.4 & 235.1 & 263.5 & 267.2 \\
\hline $\begin{array}{c}\text { Transmitted } \\
\text { Power }\end{array}$ & 0.66 & 0.73 & 0.77 & 0.76 & 0.69 \\
\hline \begin{tabular}{c}
$\theta_{3}\left[{ }^{\circ}\right]$ \\
\hline$L_{b 3}[\mu \mathrm{m}]$
\end{tabular} & 0.25 & 0.5 & 0.75 & 1.0 & 1.25 \\
\hline $\begin{array}{c}\text { Transmitted } \\
\text { Power }\end{array}$ & 0.68 & 0.76 & 0.78 & 0.75 & 0.68 \\
\hline \begin{tabular}{c} 
Powe \\
\hline
\end{tabular}
\end{tabular}

between the guided mode and radiation modes. In order to suppress the undulation, we bend the waveguide into the direction defined by the normal of the wave front at the point $P$ where the peak crosses the center axis for the first time. The normal direction $\theta_{2}$ of the wave front is given by the maximum value of the following expression [15]:

$$
f\left(z, \theta_{2}\right)=\left|\int E_{0}^{*}(x) \cdot E(x, z) \cdot \exp \left(-j \beta_{0} \sin \theta_{2} x\right) d x\right|
$$

where $E_{0}(x)$ and $\beta_{0}$ represent the transverse electric field and the propagation constant of the eigenmode of the waveguide and $E(x, z)$ does the field distribution obtained by the FDM [12]. The asterisk indicates the complex conjugate. It is expected that the optical wave undulation in the so designed bent waveguide which is shown by the solid line in Fig. 6 is suppressed in the region $i i$ and hence the radiation losses can be reduced.

\section{B. Low-Loss Design for Optical Power Dividers}

Fig. 7 shows the output side of the power divider followed by the designed bent structure with a separation $2 \mathrm{~h}$ at the output end, which is composed of the three regions $\mathrm{i}$, ii, and iii. The broken lines illustrate the simple bent waveguides whose slope is $\theta$. By applying the low-loss consideration to the regions $\mathrm{i}$ and ii for the case of $\theta_{1}=1.0^{\circ}$, the structural parameters are decided as $L_{b 1}=279.6 \mu \mathrm{m}$ and $\theta_{2}=1.525^{\circ}$. If the undulation in the region ii is suppressed completely, the same structure would be possible for the region iii. However, slight undulations still remain in the designed structure. Therefore, it is necessary to optimize the structure of the region iii for minimizing radiation losses. Table I shows the normalized transmitted powers for various slopes of the region iii and its optimum length for each slope, where $2 h=50.0$ 
TABLE II

Comparison of Transmitted Powers Among the Dividers Connected to the Multibends, S-Shaped Bends and Simple Bends

\begin{tabular}{|c|c|c|c|c|c|c|c|}
\hline \multirow{2}{*}{$\begin{array}{c}2 h \\
{[\mu m]}\end{array}$} & \multirow{2}{*}{$\begin{array}{c}L_{b} \\
{[\mu m]}\end{array}$} & \multirow{2}{*}{$\theta_{1}\left[^{\circ}\right]$} & \multirow{2}{*}{$\theta_{3}\left[^{\circ}\right]$} & \multirow{2}{*}{$\begin{array}{c}\text { Average Angle } \\
\theta \quad\left[^{\circ}\right]\end{array}$} & \multicolumn{3}{|c|}{ Normalized Transmitted Power } \\
\hline & & & & & Multi Bend & S-Shaped Bend & Simple Bend \\
\hline 50.0 & 816.8 & & & 1.122 & 0.77 & 0.68 & 0.55 \\
\hline 130.0 & 2317.4 & & & 1.384 & 0.78 & 0.93 & 0.43 \\
\hline
\end{tabular}

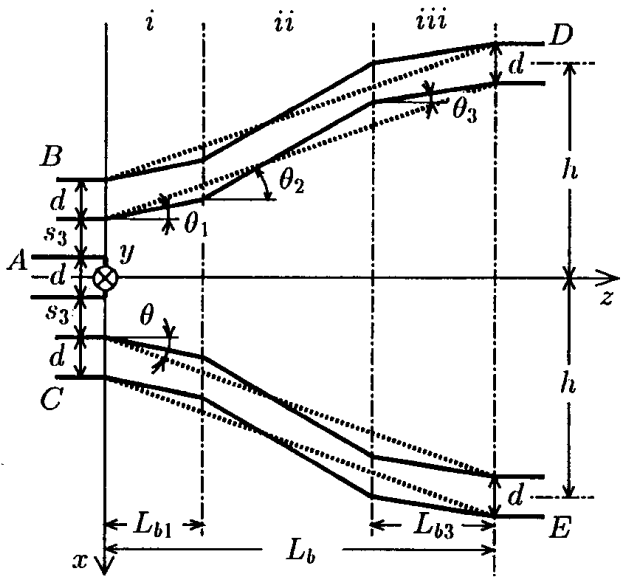

Fig. 7. Optical power dividers utilizing distributed coupling followed by bent waveguides.

$\mu \mathrm{m}$ in $(a), 2 h=130.0 \mu \mathrm{m}$ in $(b)$. As we can see, the output power takes the maximum value 0.77 for the case of $2 h=50.0$ $\mu \mathrm{m}$ with the parameters $\theta_{3}=0.75^{\circ}, L_{b 3}=235.1 \mu \mathrm{m}$, and $L_{b}=816.8 \mu \mathrm{m}$; for $2 h=130.0 \mu \mathrm{m}$, it takes 0.78 with $\theta_{3}=0.75^{\circ}, L_{b 3}=231.4 \mu \mathrm{m}$, and $L_{b}=2317.4 \mu \mathrm{m}$. The radiation losses from the distributed coupler are taken into account in these results.

So far, several low-loss bent structures have been proposed. For comparison's sake we estimate the radiation losses in the $S$-shaped bent structure whose center axis is specified by [16]

$$
W(z)= \pm\left[h_{0}+\frac{h-h_{0}}{2}\left\{1.0-\cos \left(\frac{z}{L_{b}} \pi\right)\right\}\right]
$$

where $2 h_{0}=2\left(s_{3}+d\right)$ is the separation at the output end of the distributed coupler. Table II shows the normalized transmitted powers of the $S$-shaped bends and simple bends obtained by our computing program for the FDM [12]. The normalized transmitted powers of the corresponding multi bends and their optimized structural parameters are included in the table as well. It is confirmed that the transmission characteristics of the $S$-shaped bent structure depend strikingly upon the separation or the curvature radius [16]-[18]. On the other hand, it should be noted that those of the multi bends are hardly affected by the separation. It is understood that the present multi bent structure is more suitable than the $S$-shaped bend for a narrower separation.

Fig. 8 shows the field distributions along the optical power dividers followed by the multi bends with the parameters $\theta_{1}=1.0^{\circ}, \theta_{3}=0.75^{\circ}, L_{b 1}=279.6 \mu \mathrm{m}, L_{b 3}=263.5 \mu \mathrm{m}$, and $L_{b}=816.8 \mu \mathrm{m}$ and by the simple bends with the slope

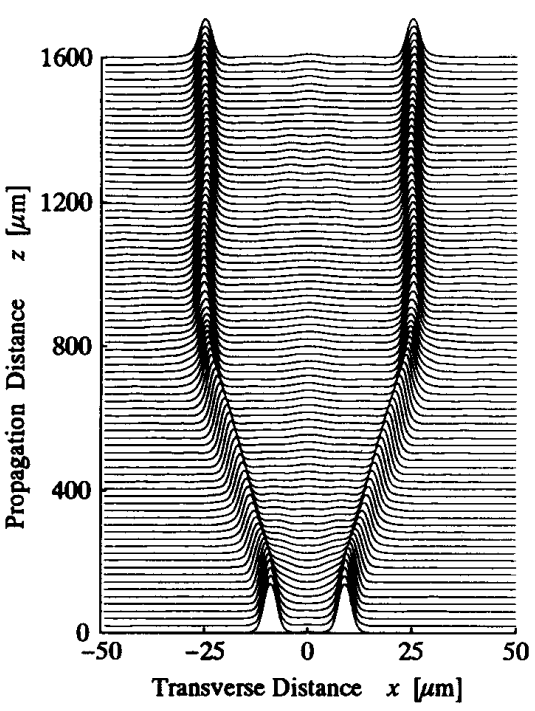

(a)

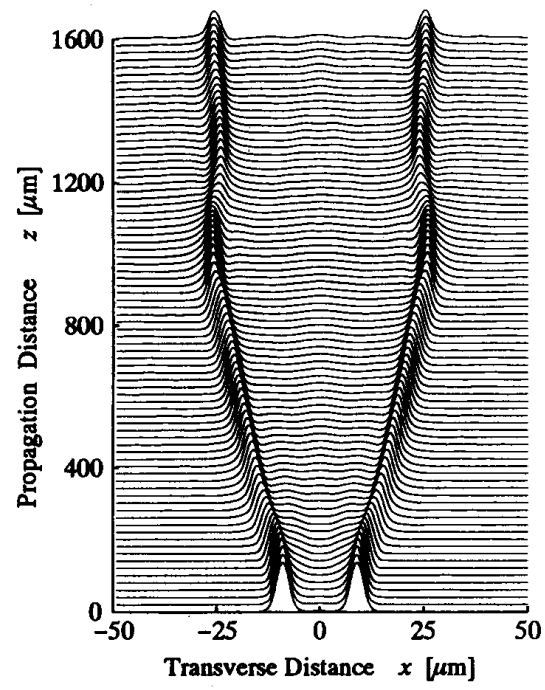

(b)

Fig. 8. Field distribution along the optical power divider followed by (a) the multibent structure with $\theta_{1}=1.0^{\circ}, \theta_{3}=0.75^{\circ}, L_{b 1}=279.6 \mu \mathrm{m}$, and $L_{b 3}=235.1 \mu \mathrm{m}$ and by (b) the simple bent structure with $\theta=1.122^{\circ}$, where $\lambda=0.6328 \mu \mathrm{m}$

$\theta=1.122^{\circ}$ where $2 h=50.0 \mu \mathrm{m}$. It is found from the figure that the optical wave undulation along the divider followed by the multi bent structure is suppressed almost completely in the region $i i$ and the output waveguides $D$ and $E$. 


\section{CONCLUSION}

In this paper, we proposed a design consideration for low-loss optical power dividers of distributed coupler type composed of three adjacent waveguides. In order to reduce the device length without changing the coupling characteristics at the output end, we introduce nonuniformly distributed coupling which is caused by step-like gap variations between the waveguides. It is found from the numerical results that, with restricting the radiation losses below $4 \%$, over a $35 \%$ reduction in the device length can be achieved in comparison with the divider utilizing an uniformly distributed coupler. Furthermore, for the purpose of obtaining wide separations at the output end, we also introduce the multi bent structure which is designed to be low-loss by the method of suppression of the optical wave undulation along the waveguide. The numerical results show that smooth separations of the optical waves without the undulation can be achieved and that over a $20 \%$ improvement in the radiation loss characteristics can be obtained in comparison with the case where the simple bent waveguides are utilized. It is also shown that the transmission characteristics of the present bent structure are stable to the change in the length of the bending section.

\section{REFERENCES}

[1] S. Sawa, M. Geshiro, and F. Takeda, "Low-loss optical branching waveguides consisting of anisotropic materials," IEEE Trans. Microwave Theory Tech., vol. 39, pp. 1140-1147, July 1991

[2] S. Sawa, K. Ono, and M. Hotta, "Mode-conversion type optical $Y$ branching waveguides fabricated in glass substrate," Trans. IEICE Jap., vol. J73-C-I, pp. 636-644, Oct. 1990.

[3] M. Hotta, M. Geshiro, and S. Sawa, "Analysis of lightwave propagation in mode-conversion-type optical $Y$-branching slab waveguides by propagation beam method," Trans. IEICE Jap., vol. J74-C-I, pp. 375-378, Oct. 1991 .

[4] K. Nakama and S. Kobayashi, "Loss reduction of ion-exchanged glass waveguide $Y$-branch," Trans. IEICE Jap., vol. J75-C-I, pp. 480-482, June 1992.

[5] T. Findarkly and B. Chen, "Single-mode integrated optical $1 \times \mathrm{N}$ star coupler," Appl. Phys. Lett., vol. 40, pp. 549-550, July 1982.

[6] J. Yamauchi, T. Ando, and H. Nakano, "Analysis of $1 \times 3$ coupledwaveguide optical power dividers by the implicit finite-difference method," Trans. IEICE Jap., vol. J75-C-I, pp. 703-710, Nov. 1992.

[7] Y. Cai, T. Mizumoto, and Y. Naito, "Analysis of coupling characteristics of a tapered three-guide coupled system," J. Lightwave Technol., vol. 8, pp. 1621-1629, Oct. 1990.

[8] M. Hotta, M. Geshiro, T. Arashiba, and S. Sawa, "Design considerations for optical power dividers of ununiformly distributed-coupling type," Trans. IEICE Jap., vol. J75-C-I, pp. 755-757, Dec. 1992

[9] M. Kaznetsov and H. A. Haus, "Radiation loss in dielectric waveguide structures by the volume current method," IEEE J. Quantum Electron., vol. QE-19, pp. 1505-1514, Oct. 1983.

[10] M. Hotta, M. Geshiro, T. Takeichi, and S. Sawa, "A design consideration for symmetric power dividers of a distributed-coupling type," IEICE Jap. Tech. Rep., vol. OQE91-161, pp. 61-66, Jan. 1992.

[11] M. Hotta, M. Geshiro, and S. Sawa, "New design consideration for low-loss single-mode bent slab waveguides by suppressing optical beam undulation," Trans. IEICE Jap., vol. J74-C-I, pp. 375-378, Oct. 1991.

[12] Y. Chung and N. Dagli, "An assessment of finite difference beam propagation method," IEEE J. Quantum Electron., vol. QE-26, pp. 1335-1339, Aug. 1990.

[13] L. M. Jonson and F. J. Leonberger, "Low-loss $\mathrm{LiNbO}_{3}$ waveguide bends with coherent coupling," Opt. Lett., vol. 8, pp. 111-113, Feb. 1983.

[14] M. D. Feit and J. A. Fleck, Jr., "Light propagation in graded-index optical fibers," Appl. Opt., vol. 17, no. 24, pp. 3990-3998, Dec. 1978.

[15] S. Kawakami and K. Baba, "Field distribution near an abrupt bend in single-mode waveguides: A simple model," Appl. Opt., vol, 24, no. 21, pp. 3643-3647, Nov. 1985.

[16] R. Baet and P. E. Lagasse, "Loss calculation and design of arbitrarily curved integrated-optic waveguide," J. Opt. Soc. Amer., vol. 73, pp.
$177-182$, Feb, 1983

[17] D. Marcuse, "Length optimization of an $S$-shaped transition between offset optical waveguides," Appl. Opt., vol. 17, no, 5, pp. 763-768, Mar. 1978.

[18] W. J. Minford, S. K. Korotky, and R. C. Alferness, "Low-loss $\mathrm{Ti}: \mathrm{LiNbO}_{3}$ waveguide bends at $\lambda=1.3 \mu \mathrm{m}$," IEEE $J$. Quantum Electron., vol. QE-18, pp. 1802-1806, Oct. 1982.

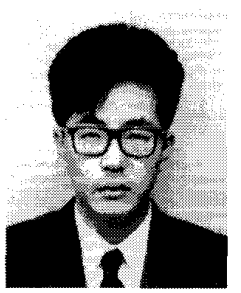

Masashi Hotta (M’92) was born August 19, 1965, in Ehime, Japan. He received the B.E. and M.E. degrees in electronics engineering form Ehime University, Ehime, Japan in 1988 and 1990 respectively.

In April 1990, he joined the Department of Electronics Engineering, Ehime University, Matsuyama, Japan, where he is now Assistant Professor of Electrical \& Electronic Engineering. He has been engaged in research and development of opto-electronic devices.

Mr. Hotta is a member of the IEICE of Japan.

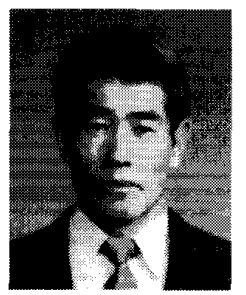

Masahiro Geshiro (S'75-M'78) was born August 28, 1949, in Osaka, Japan. He received the B.E., M.E., and Ph.D. degrees in electrical communication engineering from Osaka University, Osaka, Japan, in 1973, 1975, and 1978, respectively.

$\mathrm{He}$ is currently an Associate Professor of the Department of Electrical \& Electronic Engineering at Ehime University, Matsuyama, Japan. From March 1986 to January 1987, he was a Visiting Scholar at the University of Texas at Austin, on leave from Ehime University. He has been engaged in research on microwave and optical transmission lines and integrated circuits. Dr. Geshiro is a member of the IEICE of Japan.

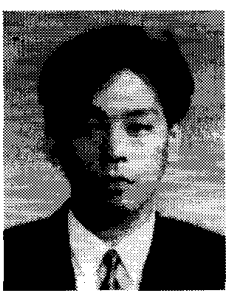

Tomoyuki Arashiba was born October 27, 1969, in Okayama, Japan. He received the B.E. degree in electronics engineering from Ehime University, Matsuyama, Japan in 1992.

$\mathrm{He}$ is presently working toward the M.E. degree in Electrical \& Electronic Engineering at Ehime University, where he has been engaged in research on optical components.

Mr. Arashiba is an associate member of the IEICE of Japan.

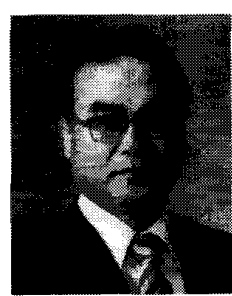

Shinnosuke Sawa (M'72) was born October 23,1938 , in Osaka, Japan. He received the B.E. degree in electrical engineering from the University of Osaka Prefecture, Osaka, Japan in 1962 and the M.E. and Ph.D. degrees in electrical communication engineering from Osaka University, Osaka, Japan, in 1967 and 1970, respectively.

From 1962 to 1964 , he worked in industry for the Mitsubishi Electric Corporation, where he was engaged in ignitron manufacture and vacuum switch development at the Kyoto plant of the Corporation. From 1970 to 1991, he was with the Department of Electronics Engineering, Ehime University, Matsuyama, Japan. Since 1991, he has been a Professor of the Department of Electrical Engineering, the University of Osaka Prefecture, Sakai, Japan, where he is engaged in research and education in electromagnetic theory, electromagnetic wave engineering, optical waveguides, and optical integrated circuit.

Dr. Sawa is a member of the IEICE of Japan, the Institute of Electrical Engineers of Japan and Laser Society of Japan. 